\title{
On the Origins of Life
}

\author{
Helen Hansma, reply by Brian Miller
}

In response to "Hot Wired" (Vol. 5, No. 2).

To the editors:

The perspective Brian Miller and Jeremy England bring to their essay on the origins of life is that of physics. Yet the origins of life are, ultimately, chemical and biological. In Miller and England's account, the biology and chemistry are weak.

"If even one enzyme were missing," Miller writes, "all metabolic processes would cease..." Not so. Some metabolic processes would cease, but many metabolic processes are independent of any specific enzyme in living cells, and no enzymes were needed at the earliest stages of life's emergence.

Picture a biomolecular ecosystem of noncellular proto-life. Autocatalytic networks of reactions occur in environments that support these reactions. Only a few of the chemically possible reactions occur. ${ }^{1}$ There are hundreds of different amino acids, but proteins in living cells contain only twenty varieties. RNA molecules evolve in isolation and grow by ligating or joining together in the presence of other RNA molecules. ${ }^{2}$ Almost everything fails. Error tolerance is a major requirement for the origins of life. ${ }^{3}$ The molecules of life accumulate and grow in tiny steps. Slowly, over millions and tens of millions of years, the molecules modify their environment and increase in complexity, becoming capable of more complex processes and the storage of more information. The prebiotic ecosystem eventually contains all the components necessary for life, but there are still no living cells. Fragments of this ecosystem are continually breaking off and moving into the external environment, often after being encapsulated in lipid membranes. Nearly all of these fragments are lacking some essential components of a living cell and are not alive. But occasionally all essential components of a living cell are enclosed in a membrane, forming a living cell. Nearly all of these living cells die, too. But not all of them.

My favorite embodiment of this ecosystem is in the spaces between mica sheets, which provide many cell- like compartments for emerging life processes. ${ }^{4}$ Between some mica sheets, there are crowded spaces where RNA molecules can combine and grow. Between other mica sheets, there are many relatively empty spaces where RNA molecules can evolve without being outcompeted by the most successful RNA-copying ribozyme. ${ }^{5}$ Molecules diffuse between the many compartments within the mica sheets. The negatively charged mica sheets have positively charged potassium ions between them, just like the cytoplasm of living cells, which have high concentrations of potassium ions. The mica sheets are crystalline, with negative charges spaced 0.5 nanometers, or 5 angstroms, apart. This is the same spacing as the phosphate groups in extended single-stranded DNA and RNA from living cells. The mica sheets are a template for building RNA and DNA with the same structure as that found in living cells. The RNA and DNA on the template of mica sheets do not have the kinks and other defects that form from incorrect linkages. The RNA and DNA have only one phosphate group, not two, between each sugar-base unit, because that is what fits on the template. The mica sheets have an endless energy source from the work of opening and closing, stretching and pressing the molecules between them.

According to Miller, moving mica sheets could not "have generated more than a tiny fraction of the required free energy." He offers no evidence to support this claim. If the mica sheets move even one angstrom closer together and have a spring constant stiff enough to provide 170 piconewtons of force, they can push together two molecules to form a covalent bond. ${ }^{6}$ The equation for a spring constant, $F=k x$, shows that a spring constant of 1.7 newton-meters would suffice. The spring constant of the mica depends on the number of mica sheets in the layer that is opening and closing. Each mica sheet is approximately one nanometer thick. Only about seven mica sheets are needed to provide this spring constant. ${ }^{7}$ Mica does indeed provide an endless energy source with more than enough energy to create the molecules of life.

"Enzymes are essential for energetically favorable reactions," Miller asserts, "since most reactions are too slow to drive cellular operations.” Not so. Enzymes are not needed as life is starting to emerge. Energetically favorable reac- 
tions will, by definition, proceed, albeit slowly. Enzymes catalyze these energetically favorable reactions. Before life existed, there was no time pressure and if the reaction proceeded, that was enough. Before life arose, the dogeat-dog world of living systems did not exist. Now there are RNases everywhere. Enzymes that degrade RNA are deposited on every surface our fingers touch.

Both authors discuss the probability of life. But life is a multistep process. Consider the probability that a fertilized egg, the fusion of egg and sperm, weighing less than one microgram, will create a living human being weighing, perhaps, three kilograms, in only nine months. The womb provides an environment in which this remarkable process can occur with a reasonable probability. Earth is the womb from which life emerged. In one or more favorable locations, molecules reacted and combined. And living cells were born.

"DNA in water falls apart spontaneously on the timescale of millions of years," England points out, "and RNA and protein degrade thousands of times faster." What living things survive for millions of years, or even thousands of years? There are a few trees and seeds of that age, but their DNA is well protected from water.

Indeed, water can be a problem for biological polymers. When they are wet, biopolymers fall apart. Amino acids polymerize to form peptides and proteins when the water dries up. But when the peptides and proteins get wet, they hydrolyze, releasing the free amino acids. ${ }^{8}$ The peptides and proteins need to shelter in a dry place, like the spaces between mica sheets, if they are to survive and grow.

"The essence of what we may most want to know about life," England remarks, "has to do with its probability." Instead, I would ask these questions: What are the conditions necessary for life? When these conditions are present on a planet, what is the probability that life could gradually emerge from the planet's womb over millions and millions of years? It happened on Earth, but has it happened on other planets?

\section{Helen Hansma}

\section{Brian Miller replies:}

In my exchange with Jeremy England, I argued that the earliest stages of life's origin required advanced molecular machinery to provide a steady supply of chemical energy and large quantities of information. ${ }^{9}$ In her letter, Helen Hansma contended that sufficient energy could have been provided by moving mica sheets. Hansma developed her mica hypothesis for the origin of life as an extension of her pioneering work on biological research with the atomic force microscope. In her response to us, she also asserts that preexistent information would not have been required since a cell could have gradually emerged from such precursors as autocatalytic chemical networks and evolving RNA molecules. In reality, the research she cites only further reinforces my thesis.

Hansma envisions that spaces between adjacent mica sheets embedded in rocks along the ocean floor could serve as the staging ground for the formation of the first cell. In her model, the relative motion of one sheet toward a neighboring one mechanically forces molecules together. Either the repulsive electrostatic force is overcome and a covalent bond is formed between the molecules, or the motion breaks them apart. The mechanical energy of the sheets is converted into chemical energy. The critical flaw in this scenario is Hansma's claim that mica might potentially provide sufficient mechanical energy for such a process. The efficiency of energy conversion in an ancient marine environment is far too low for the model to be plausible.

Numerous factors would have limited the mechanochemical energy production density-generated power per square area of mica sheet surface-to an exceedingly small value. All realistic mechanisms for synthesizing the molecular building blocks, e.g., amino acids, sugars, lipid, and metabolites, yield most as minority products in an intractable mixture with numerous other molecules. ${ }^{10}$ In addition, the different building blocks must have originated in different local environments, ${ }^{11}$ meaning that many would have needed to travel significant distances to reach the prebiotic cradle. Such a journey would have greatly diluted their concentrations. As a consequence, it seems exceedingly unlikely that the right molecules would have been able to position themselves directly opposite each other in just the right orientations against neighboring mica surfaces with just the right topologies at just the right time for the motion of the sheets to power any biochemical reaction. Just one such event occurring each second within a $1-\mathrm{cm}^{2}$ area would have been stunningly fortuitous.

In stark contrast, bacteria with all levels of complexity must generate at least 10,000 ATP molecules/second $/ \mu \mathrm{m}^{2}$ within their cell membranes to simply maintain their integrity against the constant thermodynamic forces disrupting their homeostatic stability. ${ }^{12}$ A drop in power results in metabolism halting and the cell irreversibly decomposing into an amorphous conglomeration of simpler chemicals. The required energy production rate corresponds to $1 \mathrm{~cm}^{2}$ of a mica sheet driving trillions of energetically unfavorable reactions every second.

The challenge lies in the fact that any prebiotic chemical system gradually moving away from equilibrium experiences increasingly strong thermodynamic forces pushing it back toward equilibrium..$^{13}$ In turn, the system would require an increasingly large supply of chemical energy just to counterbalance these forces and even greater amounts to continue its progress toward an autonomous cell. Mica sheets could never have supported even the earliest stages of any origin-of-life scenario.

Hansma's confidence is equally misplaced regarding the capacity of autocatalytic chemical networks and evolv- 
ing RNAs to bypass the need for preexistent information. Any evolving system of chemical processes would favor those that move the fastest towards lower free energy. In contrast, nearly every metabolic reaction within a cell does not occur spontaneously without supplied energy. If it were otherwise, the cell would be too slow to compete with countless other possible reactions in an ancient marine environment. Even if all life-relevant processes could occur as easily as abiotic processes, the chances that a random set of reactions and molecular structures coalesced into a viable cell is far too small to have ever occurred without high levels of orchestration. ${ }^{14}$

The key obstacle was described by Harold Morowitz:

Networks of synthetic pathways that are recursive and self-catalyzing are widely known in organic chemistry, but they are notorious for generating a mass of side products, which may disrupt the reaction system or simply dilute the reactants, preventing them from accumulating within a pathway. The important feature necessary for chemical selection in such a network, which remains to be demonstrated, is feedback-driven self-pruning of side reactions, resulting in a limited suite of pathways capable of concentrating reagents as metabolism does. ${ }^{15}$

The response to this challenge has been to appeal to prebiotic natural selection. Michael Russell, an architect of the hydrothermal vent origins model, remarked:

We claim in particular that it is untenable to hold that life-relevant biochemistry could have emerged in the chemical chaos produced by mass-action chemistry and chemically nonspecific "energy" inputs, and only later have evolved its dauntingly specific mechanisms (as a part of evolving all the rest of life's features). Instead, we claim, it had to have been launched simple and "specific" and thereafter have been forced by the scythe of natural selection to maintain the necessary specificity standard at each evolutionary increment in complexity. ${ }^{16}$

The obvious problem with the selection hypothesis is that prior to the emergence of self-replicating cells, natural processes would have always favored lower free-energy states, moving any chemical system away from life, rather than toward it.

Scenarios that begin with evolving RNAs are even less tenable. RNAs can only grow, combine, or replicate with the assistance of catalytic RNAs, namely ribozymes, facilitating the process. ${ }^{17}$ The likelihood that such RNAs were residing in a confined locale in sufficient concentrations to sustain RNA evolution is infinitesimal. ${ }^{18}$ Even if one assumed prolific evolution, the dominant RNA versions would quickly become those that specialize in efficient replication and consequently performed few, if any, other useful functions. ${ }^{19}$
The catalytic capacities of RNAs even under highly directed selection are too limited to serve anything but the simplest of biological functions. ${ }^{20}$ If linked to molecular cofactors, their capabilities may increase, but chance interactions with proposed candidate molecules on the early earth would have been exceptionally rare. ${ }^{21}$ At a more foundational level, RNA strands sufficiently long to perform any biochemical function could not have even emerged in non-trace quantities on the early earth, ${ }^{22}$ particularly in a marine environment. ${ }^{23}$ The probability of multiple RNAs with biologically relevant capacities converging in the same microenvironment is vanishingly small.

As discussed in my essay, the only way a complex chemical network could sustain itself far from equilibrium is if it was controlled by a highly specific set of enzymes, or their equivalent, whose amino-acid sequences contain large quantities of functional information. ${ }^{24}$ The need for information to maintain a cell's low-entropy state has been articulated by Yaşar Demirel:

The ATP synthesis, in turn, is matched and synchronized to cellular ATP utilization processes, since energy and matter flows must be directed by the information for them to be functional and serve a purpose. For living systems, the information is the cause and the entropy reduction is the result:

Energy + Matter + Information $\rightarrow$ Locally reduced entropy (Increase of order) ${ }^{25}$

Even the most conservative models of a minimally complex self-sustaining cell correspond to more than 100 proteins and more realistic analyses estimate much larger quantities. ${ }^{26}$ Multiple copies of most proteins are also required to sustain cellular operations. ${ }^{27}$ Compounding the problem, proteins have a limited lifespan, so they must constantly be replaced. They do not self-replicate, and RNAs are too unstable for long-term information storage. ${ }^{28}$ A cell's minimal requirements must therefore include the protein sequences being encoded into DNA, and the cell must possess the DNA-protein translational machinery to access the encoded information and implement it in the manufacture of new proteins. The regulatory regions of DNA are required to tightly control the quantities and timing of protein production to maintain a stable metabolism..$^{29}$ The total information requirement almost certainly exceeds a million bits.

Hansma's response in no way challenges the conclusion that the origin of life must have involved a sudden transition from a primordial chemical system into an autonomous cell, and that transition must have included the instantiation of complex energy-production machinery and a large infusion of information. Explaining such a saltation event requires a radical rethinking of the role of 
information in life's inception as suggested by Sara Walker and Paul Davies:

On practical grounds alone, we need to remain open to the possibility that the causal efficacy of information may amount to more than a mere methodological convenience, and might represent a new causal category not captured in a microstate description of the system. What we term "the hard problem of life" is the identification of the actual physical mechanism that permits information to gain causal purchase over matter. This view is not accommodated in our current approaches to physics. ${ }^{30}$

Helen Hansma is a Research Biophysicist Emeritus in the Department of Physics at the University of California, Santa Barbara.

\section{Brian Miller holds a PhD in physics from Duke University.}

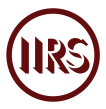

1. Aaron David Goldman, John Baross, and Ram Samudrala, "The Enzymatic and Metabolic Capabilities of Early Life," PLoS One 7, no. 9 (2012), doi:10.1371/journal.pone.0039912; Joana Xavier et al., "Autocatalytic Chemical Networks at the Origin of Metabolism," Proceedings of the Royal Society $B$ 287, no. 1,922 (2020), doi:10.1098/rspb.2019.2377.

2. Péter Szabó et al., "In Silico Simulations Reveal that Replicators with Limited Dispersal Evolve Towards Higher Efficiency and Fidelity," Nature 420, no. 6,913 (2002): 340-43, doi:10.1038/nature01187; Gerald Joyce, “Molecular Evolution: Booting Up Life," Nature 420, no. 6,913 (2002): 278-79, doi:10.1038/420278a.

3. Freeman Dyson, Origins of Life (Cambridge: Cambridge University Press, 1999), 100.

4. Helen Hansma, "Could Life Originate between Mica Sheets? Mechanochemical Biomolecular Synthesis and the Origins of Life," in Probing Mechanics at Nanoscale Dimensions, ed. Nobumichi Tamura et al. (Warrendale: Materials Research Society, 2009); Helen Hansma and Emin Oroudjev, "Atomic Force Microscopy of Biomaterials, Mica, and the Origins of Life," Microscopy Today 18, no. 6 (2010): 16-20, doi:10.1017/ S1551929510000970; Helen Hansma, "Possible Origin of Life between Mica Sheets," Journal of Theoretical Biology 266, no. 1 (2010): 175-88; Helen Hansma, "Possible Origin of Life between Mica Sheets: How Life Imitates Mica," Journal of Biomolecular Structure \& Dynamics 31, no. 8 (2013): 888-95, doi:10.1080/07391102.2012.718528; Helen Hansma, "The Power of Crowding for the Origins of Life," Origins of Life and Evolution of Biospheres 44, no. 4 (2014): 307-11, doi:10.1007/s11084-014-9382-5; Helen Hansma, "Mechanical Energy before Chemical Energy at the Origins of Life?" Sci 2 (2020): 19, doi:10.3390/sci2010002.
5. Szabó et al., "In Silico Simulations Reveal that Replicators with Limited Dispersal Evolve Towards Higher Efficiency and Fidelity”; Joyce, "Molecular Evolution: Booting Up Life."

6. Hansma, "Possible Origin of Life between Mica Sheets: How Life Imitates Mica."

7. As shown in Figure 4 of Andres Castellanos-Gomez et al., "Mechanical Properties of Freely Suspended Atomically Thin Dielectric Layers of Mica," Nano Research 5, no. 8 (2012): 550-57, doi:10.1007/s12274-012-0240-3.

8. Noam Lahav, Dawn White, and Sherwood Chang, "Peptide Formation in the Prebiotic Era: Thermal Condensation of Glycine in Fluctuating Clay Environments," Science 201, no. 4,350 (1978): 67-69, doi:10.1126/science.663639.

9. For an extended discussion, see Brian Miller, "On the Origin of Life, Here Is My Response to Jeremy England," Evolution News, May 11, 2020; Charles Thaxton et al., The Mystery of Life's Origin: The Continuing Controversy (Seattle: Discovery Institute Press, 2020), 359-74.

10. Thaxton et al., The Mystery of Life's Origin, 91-120; Alan Schwartz, “IntractableMixturesandtheOriginofLife," Chemistry and Biodiversity 4, no. 4 (2007): 656-64, doi:10.1002/ cbdv.200790056.

11. Norio Kitadai and Shigenori Maruyama, "Origins of Building Blocks of Life: A Review," Geoscience Frontiers 9, no. 4 (2018): 1,117-53, doi:10.1016/j.gsf.2017.07.007.

12. Michael Lynch and Georgi Marinov, "Membranes, Energetics, and Evolution across the Prokaryote-Eukaryote Divide," eLife 6 (March 16, 2017), doi:10.7554/eLife.20437.

13. Leonid Martyushev and Vladimir Celezneff, "Nonequilibrium Thermodynamics and Scaleinvariance," Entropy 19, no. 3 (2017), doi:10.3390/e19030126.

14. Peter Tompa and George Rose, "The Levinthal Paradox of the Interactome," Protein Science 20, no. 12 (2011): 2,074-79, doi:10.1002/pro.747.

15. James Trefil, Harold Morowitz, and Eric Smith, "The Origin of Life," American Scientist 97, no. 3 (2009): 206-13, doi:10.1511/2009.78.206.

16. Elbert Branscomb and Michael Russell, "Frankenstein or a Submarine Alkaline Vent: Who Is Responsible for Abiogenesis?" BioEssays 40, no. 8 (August 1, 2018): 1700182, doi:10.1002/bies.201700182.

17. Gerald Joyce, "Ribozymes: Building the RNA World," Current Biology 6, no. 8 (1996): 965-67, doi:10.1016/S09609822(02)00640-1. Note that protein enzymes are assumed to have originated later.

18. Piotr Bregestovski, " nario of the Origin and Early Evolution of Life on Earth," Journal of Evolutionary Biochemistry and Physiology 51, no. 1 (2015): 72-84, doi:10.1134/S0022093015010111.

19. D. R. Mills, R. L. Peterson, and S. Spiegelman, "An Extracellular Darwinian Experiment with a Self-Duplicating Nucleic Acid Molecule," Proceedings of the National Academy of Sciences 58, no. 1 (1967): 217-24, doi:10.1073/pnas.58.1.217.

20. Peter Wills, "The Generation of Meaningful Information in Molecular Systems," Philosophical Transactions of the Royal 
Society A: Mathematical, Physical and Engineering Sciences 374, no. 2063 (March 13, 2016), doi:10.1098/rsta.2015.0066.

21. David Hiller and Scott Strobel, "The Chemical Versatility of RNA," Philosophical Transactions of the Royal Society B: Biological Sciences (2011), doi:10.1098/rstb.2011.0143. The candidate molecules are much larger than nearly all comparable molecules generated in non-trace quantities from any prebiotic experiment.

22. Robert Shapiro, "A Simpler Origin for Life," Scientific American 296 (2007): 46-53, doi:10.1038/scientificamerican0607-46; James Tour, "Animadversions of a Synthetic Chemist," Inference: International Review of Science 2, no. 2 (2016).

23. Steven Benner, Hyo Joong Kim, and Matthew Carrigan, "Asphalt, Water, and the Prebiotic Synthesis of Ribose, Ribonucleosides, and RNA," Accounts of Chemical Research 45, no. 12 (2012): 2,025-34, doi:10.1021/ar200332w.

24. For a discussion on the correspondence between information and metabolic control, see Brian Miller, "The Information Enigma: A Closer Look," Evolution News, June 19, 2020. Even if the RNA world were not implausible, it would face comparable information requirements.

25. Yaşar Demirel, "Information in Biological Systems and the Fluctuation Theorem," Entropy 16, no. 4 (2014): 1,931-48, doi:10.3390/e16041931.

26. Rosario Gil et al., "Determination of the Core of a Minimal Bacterial Gene Set," Microbiology and Molecular Biology Reviews 68, no. 3 (2004): 518-37, doi:10.1128/mmbr.68.3.518537.2004 .

27. Thomas Traut, Allosteric Regulatory Enzymes (New York: Springer, 2008), 17-21.

28. Ulla Kaukinen et al., "The Reactivity of Phosphodiester Bonds within Linear Single-Stranded Oligoribonucleotides Is Strongly Dependent on the Base Sequence," Nucleic Acids Research 30, no. 2 (2002): 468-74, doi:10.1093/nar/30.2.468.

29. Indra Bervoets and Daniel Charlier, "Diversity, Versatility and Complexity of Bacterial Gene Regulation Mechanisms: Opportunities and Drawbacks for Applications in Synthetic Biology," FEMS Microbiology Reviews 43, no. 3 (2019): 304-39, doi:10.1093/femsre/fuz001; Ralf Steuer and Björn Junker, "Computational Models of Metabolism: Stability and Regulation in Metabolic Networks," Advances in Chemical Physics 142, no. 105 (2008): 110-13, doi:10.1002/9780470475935.ch3.

30. Sara Imari Walker and Paul Davies, "The 'Hard Problem' of Life," in From Matter to Life: Information and Causality, ed. George Ellis, Davies, and Walker (Cambridge: Cambridge University Press, 2017), 19-37. 